\title{
Review
}

\section{Using the 3D Ear Scanner: Seven Practical Tips to Remember if you are an Audiologist and Manage Patients with Otoscan}

\author{
Barbero Rodriguez A* \\ Audiologist, Audiocenter-Seville, Seville, Spain
}

${ }^{*}$ Correspondence to: Angel Barbero, Audiologist, Chief Coordinator of Audiocenter, Seville, Spain, Tel: + 34954989147; Email: audiocenter@audiocenter.edu.es

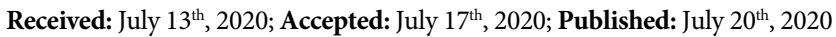

Citation: Barbero RA. Using the 3D ear scanner: Seven practical tips to remember if you are an audiologist and manage patients with otoscan. ENT Open A Open J. 2020; I(1): 1-3.

\begin{abstract}
Otoscanner 3D is a new technique used to reproduce the shape and measurements of patients' ears in Audiology and Anaplastology, using laser light beams and 3D modeling. This new technique is increasingly used by audiologists because it has high precision and the great advantage of making a digital design of the molds personalized with the patient's anatomy. In a very short time, it has become equipment for daily use in Audiology Clinics and Anaplastology. Our audiologists periodically review the protocols for using the 3D scanners and provide useful advice and corrections for their colleagues in their daily work with the 3D scanning systems. To know the complete evolution of our work, see the two articles previously published in industry magazines and in professional forums and which are referenced in the article. ${ }^{1-3}$ Manufacturers edit a manual with the use of the Otoscanner that the audiologist should know, but normally the advice about the difficulties we have during daily practice with $3 \mathrm{D}$ scanning and when working with difficult patients during the measurement is not edited. To serve as a guide, we publish here some of those aspects that include correcting the patient's position to perform the measurement; advantages of using in combination with the Video-Otoscope; advice if you also take measures for Anaplastology; precautions to consider such as restrictions on use below adulthood; improvements in your hardware; avoid saturation of the Otocloud and Otoscan-COVID.
\end{abstract}

Keywords: Otoscan 3D; Ear Scanner; Digital Ear Scanning; Anaplastology; Earmoulds; Ear measurements in Audiology.

\section{REVIEW}

\section{Legal Warning-Transparency Law (EEC)}

This article is not paid with subsidies or public company funds. And it is an independent and neutral source of opinion, without commercial influence or intention.

This work is a summary of our experience in ear scanning using $3 \mathrm{D}$ techniques in recent months, from our previous article ${ }^{1}$ to the present moment (Summer 2020 post-COVID). This update attempts to provide useful advice for audiologists working with Otoscanner; and it started when the first Otoscan 3D systems were installed in the country. ${ }^{2,3}$ It is not sponsored by any manufacturer and is an independent and periodic work, without commercial influences. We show the Audiologist's perspective during his daily work and the difficulties we have when working with patients in the scanner and how we think that it can be improved by giving a summary of the guidelines for the professionals of the union.

\section{About the Position of the Patient's Head When Taking Measurements}

The Otoscanner 3D units have reference coordinates fixed on the measuring headband (Figure 1), whose function is that the cameras provide the system with information at all times about the position in which they are, both the measuring gun and the patient's head. In this way, the variations produced by the rotation of the technician's hand, the slight movements of the patient or changes in verticality, would not affect the mapping. In addition, the Otoscan has at the end of the test a verification of the horizontal axis to align the microphones, if the use of the mapping is for hearing aid. That is, theoretically, the posture in which the patient sits in the examination chair does not influence. The scanner should always generate a correct digital mapping, which also has the reference of the horizontal axis. Assuming that the measurement is performed in an upright position at $90^{\circ}$, which is the recommended normal use of the prosthesis during the day. 
Figure 1. Hoop headband Otoscan.

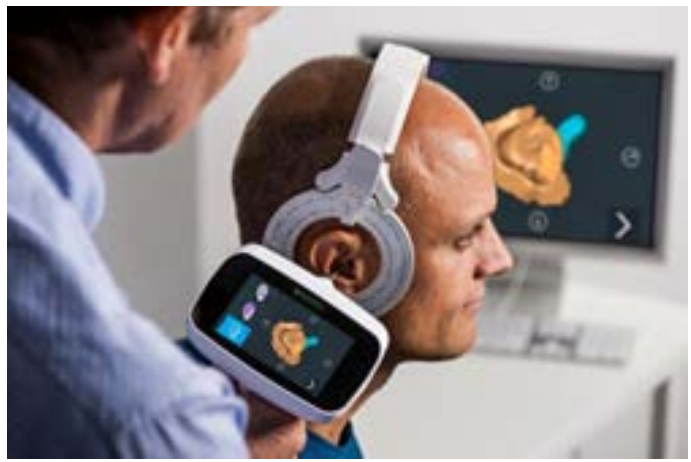

But not exactly like this. In our experience, when we scan the patient's ear, the three positions in which we obtain the best results are:

(TIP A) For adults over 70-75 years: With the chair reclined at $120^{\circ}$ (Figure 2). This gives the elderly patient a better feeling of comfort and rest than when sitting in an upright position at $90^{\circ}$. They stay more relaxed and move less, making the measurement more accurate. Furthermore, the audiologist is not in a forced position for the movements of the measuring gun. Remember to return to the vertical upright position (without losing the reference of the headband) when finishing and marking the true horizontal of use. Otherwise, it will drive your lab technician (and the system) crazy.

Figure 2. Chair reclined at $120^{\circ}$

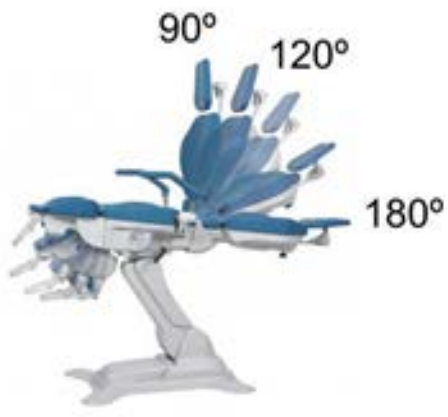

(TIP B) For young adults: with the chair at $90^{\circ}$ and their head resting on the chair (Figure 3). In this case, the patient does not have difficulties to remain still in an upright position at $90^{\circ}$. Young adults are more collaborative and tend to remain more static. Being vertical the measurement is easier for the audiologist, the scanning sweep is smoother and cleaner and the result more accurate and without "artifacts".

Figure 3. Head resting on the chair.

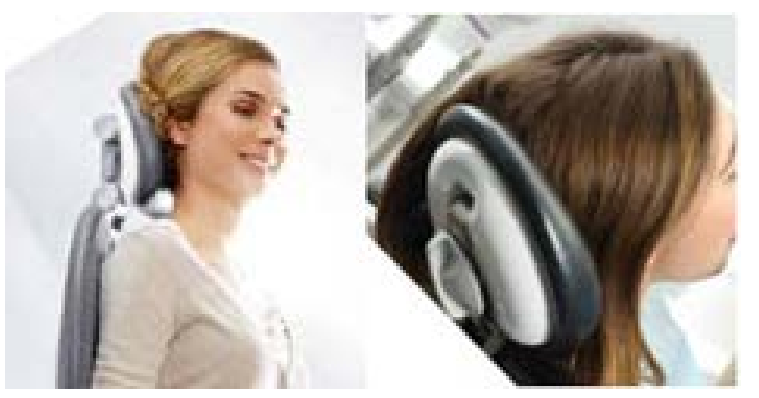

(TIP C) For all ages: In all cases, raise the patient's chair a little more than usual to make it easier for the scanner cameras to be in the most frontal position possible with respect to the headband and less inclined, so they "see" better the references. You will get greater precision in your measurements. Note that if the cameras lose the headband references, the scanning and scanning sound stops.

(TIP D) If the audiologist usually performs the otoscopic examination with a microscope and the patient lying on the stretcher: Audiologist sometimes has a tendency to take the to scanner measurements in that position (with stretcher) and then sit the patient upright to fix the true horizontal position. Our advice is NOT to use the patient's examination chair in the $180^{\circ}$ position, decubitus supine (Figure 4 ). Because, unlike the microscope examination in which $180^{\circ}$ works better, in the case of the Otoscanner 3D the result is worse. This is because the twist of the wrist of the technician's hand with the pistol scanner makes continuous scanning more difficult. When using inclinations greater than $120^{\circ}$ the curves of the external auditory canal, especially on the contralateral side to the dominance of the technician's hand (righthanded or left-handed), greatly hinder the precision of the measurement. Even if then we sit the patient to fix the true horizontality.

Figure 4. Decubitus supine-Not recomended.

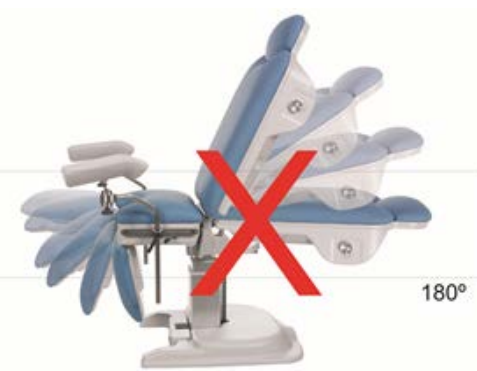

\section{Joint Use of the Videostoscope with the Otoscanner}

(TIP E) Any manipulation inside the ear canal can cause annoying sensations in very sensitive patients. Remember that. You can carry out a previous video otoscopy as a test(it will allow you to test the reactions that the patient will have with the subsequent manipulation due to the measurement of the ear scanner3D).

Before any audiology test, the test must be explained in detail to the patient, of course. But even so, there are always fears in patients due to their ignorance about what we are going to do with them. For this reason, it is also convenient to carry out a VIDEO-Otoscopy, instead of doing it with a traditional manual otoscope (Figure 5). In this way, we not only obtain the necessary prior otoscopic information, but also provide the patient with a similar feeling (as a pre-trial) of what we are doing exactly, and how we are going to do it and what is expected of him. And so when we go to the 3D scanning phase (which requires more time and precision) the patient will be used to it and will collaborate better

Figure 5. Viedo-Otoscope $v s$ Hand Otoscope

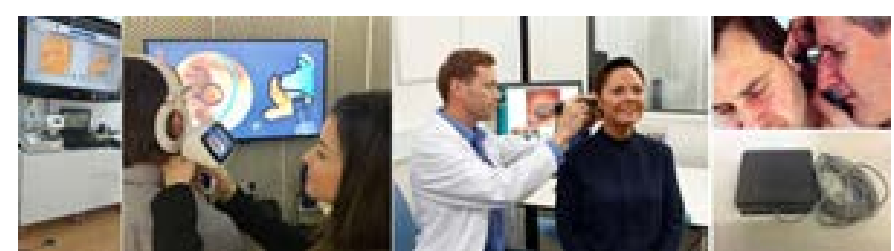


The video-otoscope also has another advantage: capturing the image displayed on the same 42-inch screen is done using the capture/ freeze footswitch (and its ease of disposal in case of failure, simply step on again the foot button).

\section{Measurements for Anaplastology}

(TIP F) Although only the inner part of the ear canal is needed in audiology, we recommend that you scan the entire ear, both inside and outside (Figure 6). This allows us to improve the technician's ability to scan, and in this way when we perform the scan in order to use it for Anaplastology, we will have acquired the ability to reproduce the entire anatomy. On the other hand, when the purpose is for use in Audiology, we can show the patient the whole of his ear on the screen, which allows him to better understand what he is seeing and how the hearing aid placed on his own ear will later be (remember that the appearance of the interior of the duct is somewhat abstract for a patient). In addition, this also allows the laboratory technician to have abundant anatomical references when designing the most appropriate mold (total size of the ear, orientation, morphological anomalies, ears separated from the head, etc.)

Figure 6. Entire ear (outside $v s$ inside).

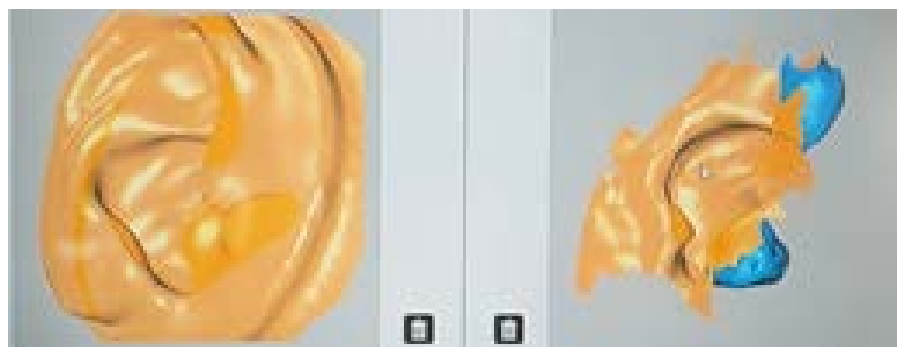

(TIP G) Precautions Remember that, just like using an otoscope, if the patient is scared, he can make an involuntary movement of the head and collide with the rigid tip of the Otoscanner 3D.

Although in theory, there are few cases of discomfort due to laser light bursts (epileptic), there are more cases than "a priori" we imagine.

\section{Application in Patients Under 16}

The manufacturer recommends scanning from young teens (16 years of age or older). Although this may be an excessive precaution, it is based on a derivation of the legal responsibility of the manufacturer itself. It will depend on the country where you practice, the health regulations, and your own experience. In this sense, it should be noted that currently scanning in children is impossible due to the diameter of the tip. It is worth remembering the same thing we asked ourselves in previous articles: for when a truly pediatric system?

Hardware: The difficulty in processing the files when we scan the entire ear (outer + inner) is much greater, due to a large number of points to be mapped. This forces the computer to work harder and sometimes it crashes and freezes. To avoid this, the manufacturer recommends the use of the computer that delivers in dedicated mode only to the Otoscan3D. Despite this difficulty, we prefer the full ear scan. But we do suggest that if you are installing/acquiring equipment of this type, ask that it be with a computer with a higher power level than the standard one, it will be very useful for a low cost.

Do not saturate your Otocloud: Many of the measurements made are not finally necessary. Otocloud is supposed to be infinite, but don't saturate it. Discard unnecessary measures and upload only the essential ones, in the long run, it will make your job easier. We currently only leave the essentials.

To finish: Otoscan and COVID. Pay special attention to cleaning the complete system: the scanning gun, the headband, cables, calibration support, etc. and value disinfection using U.V light, in addition to chemical disinfection. We can't make any recommendations on this topic yet (perhaps in the next review), because it's too recent.

But prepare ALL possible precautionary measures. As in all other audiology measurements: Don't skimp on this.

\section{REFERENCES}

1. Rodriguez AB, Salas MM, Roldan LP. What Did We Learn About OTOSCAN3D During 2019?. Int J Rhinol Otol. 2019; (1):105.

2. Barbero A. State of the Art and Future Trends on the Measurements of the Patient's Ear in the Audiology and Otologic Anaplastology Implementation of New Technologies of "3D Inside-the-Canal Auditory Scanners”. Otolaryngol (Sunnyvale). 2019; 9: 369. doi: 10.4172/2161119X.1000369.

3. Audiocenter-Sevilla: Informe Roadshow Spring-Summer 2019: Analysis \& Data, LinkediIn review, published July 16, 2019. 